\title{
THE PROBLEM OF A RIGID PUNCH MOVING ON A VISCOELASTIC HALF-PLANE WITH INERTIAL EFFECTS APPROXIMATELY INCLUDED
}

\author{
J. M. GOLDEN
}

(Received 30 June 1978)

\begin{abstract}
The problem of an infinitely long rigid punch of uniform cross-section moving across a viscoelastic half-space at constant velocity, large enough so that inertial effects cannot be neglected, is examined and solved in various approximations. Frictional shear is assumed to exist between the punch and the half-space. The method, which is an extension of that developed in previous papers $[6,7]$, is applicable for any form of viscoelastic behaviour in the half-space. For the special case of discrete spectrum behaviour the method is described in detail. For the case where the punch is cylindrical and viscoelastic effects are small compared with elastic effects, explicit expressions are given for all quantities of interest, in particular the coefficient of hysteretic friction. A general Hilbert transform formula is derived in the appendix.
\end{abstract}

\section{Introduction}

In two recent papers $[6,7]$ a method was developed for solving the problem of an infinitely long punch of uniform cross-section moving on a viscoelastic half-space $\dagger$ without special assumptions about the nature of the viscoelastic behaviour. The method applies both to the case where Coulomb frictional shear exists between the punch and the half-plane [7] and to the completely lubricated case [6]. However, it was assumed in these papers that the velocity of motion was sufficiently low to allow inertial effects to be neglected. The object of this paper is to extend the method so that this assumption may be dropped.

In Section 2 a singular integral equation is derived relating displacement and pressure, which is a generalization of similar equations given in $[6,7]$. This is achieved by virtue of the analogy between the Fourier transformed viscoelastic

$\dagger$ Often termed half-plane in this context. 
equations and the corresponding elastic equations, which makes it possible to take over some results of Eason [1] almost without modification. In its exact form little further progress can be made with this equation. However, it is shown that if certain types of approximation are made then the methods of $[6,7]$ can be applied here with little modification. Two distinct types of approximation are considered: (a) an assumption that the velocity is sufficiently small so that a power series expansion in terms of its ratio to the speeds of sound of the medium converges rapidly, and (b) an assumption that viscoelastic effects are small compared with elastic effects. In case (a) it is shown that the problem can be reduced to the solution of a certain series of non-singular integral equations, rather than just one, as was the case in previous work [6,7]. As in previous papers, the resulting expressions for quantities of interest are highly implicit, requiring considerable numerical work to obtain final answers. An advantage of the second type of approximation, which can be handled similarly though more simply, is that the results are completely explicit and easy to evaluate numerically. Also, there is no restriction to low velocities.

In the previous papers the assumption was made that the half-plane behaves similarly in shear and bulk deformation so that a unique Poisson's ratio can be defined. This assumption was not strictly necessary, but was made in the interests of simplicity. The same assumption will be made here at a certain stage when considering the approximation of type (a) but not when making the type (b) approximation. The analysis is confined to smooth punch shapes not because the method is not more generally applicable but because the resulting integrals are difficult to handle, at least for spectrum models.

The approach under the type (a) assumption of small velocity is discussed in general in Section 3 to first order in the velocity expansion. The results for the special case where the half-space viscoelasticity is characterized by a discrete spectrum is presented in detail in Section 4 while in Section 5 the assumption is made that the punch has cylindrical shape and even more detailed formulae are given. These two sections illustrate the use of the general method. The results under the type (b) small viscoelasticity assumption are presented in Section 6, together with numerical examples, and some concluding remarks in Section 7. A relevant Hilbert transform, evaluated in [7], is discussed from a different angle in the Appendix. It should be remarked that many of the results and even the presentation closely parallel that of [7], the results of which are frequently used.

\section{Derivation of basic equations}

Partly to establish notational conventions certain basic relations will be written down. The constitutive equations of a linear, homogeneous, isotropic, viscoelastic 
material may be written as

$$
\sigma_{i j}(\mathbf{R}, t)=2 \int_{-\infty}^{\infty} \mu\left(t-t^{\prime}\right) \varepsilon_{i j}\left(\mathbf{R}, t^{\prime}\right) d t^{\prime}+\delta_{i j} \int_{-\infty}^{\infty} \lambda\left(t-t^{\prime}\right) \varepsilon_{k k}\left(\mathbf{R}, t^{\prime}\right) d t^{\prime}
$$

where $\sigma_{i j}(\mathbf{R}, t), \varepsilon_{i j}(\mathbf{R}, t)$ are the components of the stress and strain tensors respectively at position $\mathbf{R}$, denoting the vector $(X, Y, Z)$, and time $t$. The summation convention is in force in the second term. The functions $\mu(t), \lambda(t)$ are closeiy related to the shear and bulk relaxation functions [6]. $\dagger$ They are both zero for negative $t$. The inverse relation may be written as

$$
\varepsilon_{i j}(\mathbf{R}, t)=\frac{1}{2} \int_{-\infty}^{\infty} \gamma\left(t-t^{\prime}\right) \sigma_{i j}\left(\mathbf{R}, t^{\prime}\right) d t^{\prime}+\delta_{i j} \int_{-\infty}^{\infty} \psi\left(t-t^{\prime}\right) \sigma_{k k}\left(\mathbf{R}, t^{\prime}\right) d t^{\prime},
$$

where $\gamma(t), \psi(t)$ are similarly related to the creep functions and zero for negative $t$. The Fourier transform (often abbreviated to FT henceforth), with respect to time, of these equations reads

$$
\begin{aligned}
& \bar{\sigma}_{i j}(\mathbf{R}, \omega)=2 \bar{\mu}(\omega) \varepsilon_{i j}(\mathbf{R}, \omega)+\delta_{i j} \bar{\lambda}(\omega) \varepsilon_{k k}(\mathbf{R}, \omega), \\
& \bar{\varepsilon}_{i j}(\mathbf{R}, \omega)=\frac{1}{2} \bar{\gamma}(\omega) \bar{\sigma}_{i j}(\mathbf{R}, \omega)+\delta_{i j} \bar{\psi}(\omega) \sigma_{k k}(\mathbf{R}, \omega),
\end{aligned}
$$

in the notation

$$
\tilde{f}(\omega)=\int_{-\infty}^{\infty} f(t) \exp (-i \omega t) d t .
$$

The quantities $\bar{\mu}(\omega), \bar{\lambda}(\omega)$ are closely related to $\bar{\gamma}(\omega), \bar{\psi}(\omega)$. Specifically

$$
\begin{aligned}
\bar{\mu}(\omega) & =1 / \bar{\gamma}(\omega), \\
2 \bar{\mu}(\omega)+3 \bar{\lambda}(\omega) & =\left(\frac{1}{2} \bar{\gamma}(\omega)+3 \bar{\psi}(\omega)\right)^{-1} .
\end{aligned}
$$

The relation (2.5) is the FT version of [6].

$$
\int_{0}^{t} \mu(t-s) \gamma(s) d s=\delta(t)
$$

where $\delta(t)$ is the usual delta function.

Note the well-known correspondence between (2.3) and the constitutive equations for a linear isotopic homogeneous elastic medium, or more precisely the FT of these equations. The difference is that here the moduli are in general frequency dependent and complex.

The equations of motion have the standard form

$$
\frac{\partial}{\partial x_{j}} \sigma_{i j}(\mathbf{R}, t)=\rho \dot{U}_{i}(\mathbf{R}, t)
$$

† The quantities $\mu(t), \gamma(t)$ as defined here differ from those in [6] by factors of $2, \frac{1}{2}$, respectively. They agree with those in [7]. 
where $\rho$ is the density of the medium and $U_{i}(\mathbf{R}, t)$ are the components of displacement. The dots indicate time differentiation. The time FT of these equations reads

$$
\frac{\partial}{\partial x_{j}} \bar{\sigma}_{i j}(\mathbf{R}, \omega)=-\omega^{2} \rho \bar{U}_{i}(\mathbf{R}, \omega)
$$

which once more has a form identical to the corresponding FT elastic equations. This identity will form the basis of our method of attacking the problem.

Let the half-space occupy $Z \leqslant 0$. Let a rigid punch be pressed into it and moving across it in the negative $X$ direction with speed $V$. Frictional shear between the plane and the punch will act along the $X$ direction. For the moment no assumption will be made on the shape of the punch.

The elastic half-space problem has been tackled, using FT techniques, by Eason [1], among others. In particular, he writes down equations relating the FT of the displacements to the FT of the surface forces. The $Z$ component of these equations may be regarded as the FT of the Boussinesq-Cerruti equation [9] generalized to include inertial effects. The difficulty is that it is in general not possible to obtain an explicit inverse FT of this equation. However, it will be taken over more or less intact and will form the basis for attacking the present problem, for which progress is possible partly because of the assumption that the punch is uniformly infinite in one direction, and partly as a result of certain approximations which will be made.

Let the pressure and frictional shear due to the punch be denoted by $P(X, Y, t)$, $S(X, Y, t)$. These will of course be non-zero only in the region of contact. Eason's equations involve not only the time FT but also the space FT of these quantities and of the displacements. To avoid excessive notational complications, the same bar notation will be used to indicate the triple FT:

$$
\tilde{P}(\mathbf{k}, \omega)=\int_{-\infty}^{\infty} P(X, Y, t) \exp \left[-i\left(k_{x} X+k_{y} Y+\omega t\right)\right] d x d y d t
$$

where $\mathbf{k}$ denotes the two dimensional vector $\left(k_{x}, k_{y}\right)$, and so on. One may distinguish between this and the time FT by the arguments. The only displacement of interest here is the $Z$ displacement at the surface. This will be denoted by $U(X, Y, t)$. Then, Eason's equation reads, with the conventions of this paper,

$$
\begin{array}{r}
2 \tilde{\mu}(\omega) F(k, \omega) \bar{U}(\mathbf{k}, \omega)=\frac{1}{2} \frac{\omega^{2}}{c_{T}^{2}(\omega)} n_{1}(k, \omega) \bar{P}(\mathbf{k}, \omega)-i k_{x} \bar{S}(\mathbf{k}, \omega) \\
\times\left(n_{1}(k, \omega) n_{2}(k, \omega)-k^{2}+\frac{1}{2} \frac{\omega^{2}}{c_{T}^{2}(\omega)}\right),
\end{array}
$$


where $k$ is the magnitude of $\mathbf{k}$, while

$$
\begin{aligned}
& n_{1}(k, \omega)=\left(k^{2}-\frac{\omega^{2}}{c_{L}^{2}(\omega)}\right)^{t} \\
& n_{2}(k, \omega)=\left(k^{2}-\frac{\omega^{2}}{c_{T}^{2}(\omega)}\right)^{\frac{1}{2}}
\end{aligned}
$$

the quantities $c_{T}(\omega), c_{L}(\omega)$ being the complex transverse and longitudinal frequencydependent wave speeds for the medium, given by

$$
\begin{aligned}
c_{T}^{2}(\omega) & =\frac{\bar{\mu}(\omega)}{\rho}, \\
c_{L}^{2}(\omega) & =\frac{\bar{\lambda}(\omega)+2 \bar{\mu}(\omega)}{\rho} .
\end{aligned}
$$

Finally, $F(k, \omega)$ is given by

$$
F(k, \omega)=\left(k^{2}-\frac{1}{2} \frac{\omega^{2}}{c_{T}^{2}(\omega)}\right)^{2}-n_{1}(k, \omega) n_{2}(k, \omega) k^{2} .
$$

For uniform motion, neglecting transients, the stresses and displacements will depend on $X+V t$ rather than on $X, t$ separately. This leads to a factor $\delta\left(w-k_{x} V\right)$ on both sides of (2.11) giving

$$
\begin{aligned}
& 2 \bar{\mu}\left(V k_{x}\right) G(\mathbf{k}) \bar{U}(\mathbf{k})=\frac{1}{2} k_{x}^{2} \delta_{T}\left(k_{x}\right) m_{L}(\mathbf{k}) \bar{P}(\mathbf{k})-i k_{x} \bar{S}(\mathbf{k}) \\
& \times\left(m_{L}(\mathbf{k}) m_{T}(\mathbf{k})-k_{x}^{2}\left(1-\frac{1}{2} \delta_{T}\left(k_{x}\right)\right)-k_{y}^{2}\right),
\end{aligned}
$$

where

$$
\begin{aligned}
& \delta_{T}\left(k_{x}\right)=\frac{V^{2}}{c_{T}^{2}\left(V k_{x}\right)}, \\
& \delta_{L}\left(k_{x}\right)=\frac{V^{2}}{c_{L}^{2}\left(V k_{x}\right)},
\end{aligned}
$$

while

$$
\begin{aligned}
m_{L}(\mathbf{k}) & =\left(k_{x}^{2}\left(1-\delta_{L}\right)+k_{y}^{2}\right)^{\frac{1}{2}}, \\
m_{T}(\mathbf{k}) & =\left(k_{x}^{2}\left(1-\delta_{T}\right)+k_{y}^{2}\right)^{\frac{1}{2}}, \\
G(\mathbf{k}) & =\left(k_{x}^{2}\left(1-\frac{1}{2} \delta_{T}\right)+k_{y}^{2}\right)^{2}-m_{L}(k) m_{T}(k) k^{2} .
\end{aligned}
$$

The final simplification is to restrict ourselves to plane problems. We let the rigid punch be infinite with uniform cross-section in the $Y$ direction. The $Y$ dependence of all quantities disappears, giving a factor $\delta\left(k_{y}\right)$ in the FT quantities.Therefore, 
under this assumption, (2.15) simplifies to

$$
2 \bar{\mu}\left(V k_{x}\right) \Gamma_{1}\left(k_{x}\right) k_{x} \bar{U}\left(k_{x}\right)=\Gamma_{2}\left(k_{x}\right) \tilde{P}\left(k_{x}\right) \operatorname{sgn}\left(k_{x}\right)-i \Gamma_{3}\left(k_{x}\right) \bar{S}\left(k_{x}\right),
$$

where $\operatorname{sgn}\left(k_{x}\right)$ is the usual sign function and

$$
\begin{aligned}
& \Gamma_{1}\left(k_{x}\right)=A_{3}^{2}-A_{1} A_{2}, \\
& \Gamma_{2}\left(k_{x}\right)=\frac{1}{2} \delta_{T}\left(k_{x}\right) A_{1}, \\
& \Gamma_{3}\left(k_{x}\right)=-A_{3}+A_{1} A_{2},
\end{aligned}
$$

the quantities $A_{i}, i=1,2,3$, being defined by

$$
\begin{aligned}
& A_{1}=\left(1-\delta_{L}\right)^{\frac{1}{2}}, \\
& A_{2}=\left(1-\delta_{T}\right)^{\frac{1}{2}}, \\
& A_{3}=1-\frac{1}{2} \delta_{T},
\end{aligned}
$$

where the explicit $k_{x}$ argument has been omitted. It remains to take the inverse transform of (2.18). We divide across by $\Gamma_{2}\left(k_{x}\right)$ so the relevant quantities are

$$
\begin{aligned}
& K_{1}(t)=\frac{1}{2 \pi} \int_{-\infty}^{\infty} \exp (i \omega t)\left[\frac{2 \bar{\mu}(\omega) \Gamma_{1}(\omega / V)}{\Gamma_{2}(\omega / V)}\right] d \omega, \\
& K_{2}(t)=\frac{1}{2 \pi} \int_{-\infty}^{\infty} \exp (i \omega t)\left[\frac{\Gamma_{3}(\omega / V)}{\Gamma_{2}(\omega / V)}\right] d \omega .
\end{aligned}
$$

The argument of these functions is of course time, and integrals involving these functions are really integrals over past history. By arguments based on causality therefore, one can see that $K_{1}(t), K_{2}(t)$ must be zero for negative $t$ just as is the case for the viscoelastic functions.

Using the result

$$
\frac{1}{2 \pi} \int_{-\infty}^{\infty} \operatorname{sgn}\left(k_{x}\right) \exp \left(i k_{x} X\right) d k_{x}=\frac{i}{\pi X}
$$

and the convolution theorem [10], one finally obtains

$$
\begin{aligned}
\frac{1}{V} \int_{-\infty}^{X} K_{1}\left(\frac{X-Y}{V}\right) U^{\prime}(Y) d Y= & \frac{1}{\pi}(\mathrm{P}) \int_{a}^{b} \frac{P(Y)}{Y-X} d Y \\
& +\frac{1}{V} \int_{a}^{X} K_{2}\left(\frac{X-Y}{V}\right) S(Y) d Y, \quad X \in[a, b] \\
= & \frac{1}{\pi} \int_{a}^{b} \frac{P(Y)}{Y-X} d Y, \quad X<a,
\end{aligned}
$$


where $[a, b]$ is the contact region, the prime denotes differentiation, and $(\mathrm{P})$ denotes the principal value of the integral. This is the general result on which subsequent considerations will be based. It is convenient to express it in terms of dimensionless coordinates defined by

$$
x=\frac{2 X-(b+a)}{b+a}
$$

putting

$$
\begin{gathered}
u^{\prime}(x) \equiv U^{\prime}(X), \\
p(x) \equiv P(X), \\
s(x) \equiv S(X),
\end{gathered}
$$

and also

$$
k_{i}(x) \equiv \sigma K_{i}(\sigma x)
$$

where

$$
\sigma=\frac{b-a}{2 V}
$$

Instead of the viscoelastic functions $\mu(t), \gamma(t)$ and so on, the quantities

$$
\begin{aligned}
& \mu_{T}(x) \equiv \sigma \mu(\sigma x), \\
& \gamma_{T}(x) \equiv \sigma \gamma(\sigma x),
\end{aligned}
$$

and so on, will often be used. The friction force between the punch and the halfspace will be taken to be given by Coulomb's Law, that is

$$
s(y)=f p(y),
$$

where $f$ is a constant, namely the coefficient of friction. Therefore, our final equation reads

$$
\begin{aligned}
\int_{-\infty}^{x} k_{1}(x-y) u^{\prime}(y) d y & =\frac{1}{\pi}(\mathrm{P}) \int_{-1}^{1} \frac{p(y)}{y-x} d y+f \int_{-1}^{x} k_{2}(x-y) p(y) d y, \quad x \in[-1,1] \\
& =\frac{1}{\pi} \int_{-1}^{1} \frac{p(y)}{y-x} d y, \quad x<-1
\end{aligned}
$$

In the limit $\delta_{T}$ or $\delta_{L}$ tending to zero $k_{2}(x)$ reduces to a delta function if the material behaves similarly in shear and bulk; in other words, if a unique, constant Poisson's ratio $v$ exists

$$
k_{2}(x)=-\beta \delta(x)+\frac{1}{\eta} m_{2}(x)
$$


where

$$
\beta=\frac{\delta_{L}}{\delta_{T}}=\frac{c_{T}^{2}}{c_{L}^{2}}=\frac{1-2 \nu}{2(1-\nu)}
$$

(which is not the sense in which it is used in [1]), and $\eta$ is a factor introduced for later convenience, given by

$$
\eta=1-\nu
$$

Note that if the material does not behave similarly in shear and bulk then, even in the non-inertial limit, the rightmost term of (2.30) remains an integral. As will be seen below, this means that the method of [7] does not trivially generalize to this case. In the absence of friction, however, there is no problem in principle if the constant Poisson's ratio assumption is dropped.

The function $m_{2}(x)$ contains specifically inertial effects and is proportional to $\delta_{T}$. Equation (2.31) also holds in the limit of small viscoelasticity but in this case no assumption on similar behaviour in bulk and shear is necessary. This follows from (2.21). It is convenient to separate out the delta function explicitly from $k_{2}(x)$ and write (2.30) in a form similar to the corresponding equation in [7]:

$$
\begin{aligned}
W(x) & =-\frac{\eta}{\pi}(\mathbf{P}) \int_{-1}^{1} \frac{p(y) d y}{y-x}+c p(x), \quad x \in[-1,1], \\
& =-\frac{\eta}{\pi} \int_{-1}^{1} \frac{p(y) d y}{y-x}, \quad x<-1,
\end{aligned}
$$

where

$$
\begin{gathered}
c=f \beta \eta=\left(\frac{1}{2}-\nu\right) f \\
W(x)=\int_{-\infty}^{x} m_{1}(x-y) u^{\prime}(y) d y+f \int_{-1}^{x} m_{2}(x-y) p(y) d y, \quad x \in[-1,1] \\
=\int_{-\infty}^{x} m_{1}(x-y) u^{\prime}(y) d y, \quad x<-1
\end{gathered}
$$

where

$$
m_{1}(x)=-\eta k_{1}(x)
$$

There are two interconnected obstacles to handling (2.34) in a manner similar to that used in [7]. In the first place there is in general no question of obtaining exact explicit expressions for $m_{i}(x), i=1,2$, except perhaps for very special forms of the viscoelastic functions. Any such specific forms will not be sought. Instead, two distinct types of approximation will be investigated which enable one to calculate $m_{i}(x), i=1,2$, and solve (2.34) for general classes of viscoelastic functions. These 
approximations are (a) to take $V$ sufficiently small so that an expansion of $m_{i}(x)$, $i=1,2$, in powers of $V$ converges rapidly, or (b) to assume that viscoelastic effects are much smaller than elastic effects and solve (2.33) to first order in viscoelastic effects. Either of these assumptions allows progress to be made, though the latter will allow far more explicit final results to be derived than the former. In fact the solution of the problem under the type (a) assumption is analogous to, though more complicated than, the exact treatment of the problem in $[6,7]$, while the solution under type (b) is very similar to the small viscoelasticity solution also presented in those papers.

The other problem is the second term on the right of the first equation of (2.36), which at first sight makes it impossible to apply the methods of [7]. However, this problem is avoided by the fact that it is anyway necessary to seek approximate solutions of the type mentioned in the previous paragraph, that is power series in an expansion parameter $\alpha$, which can be either a velocity variable, $\delta_{T}$ for example, or some number characterizing the order of magnitude of the viscoelastic coefficients. Then, if $p_{n}(x), u_{n}^{\prime}(x)$ are approximations to $p(x), u^{\prime}(x)$ of order $\alpha^{n}$, the $n$th approximation to

$$
W_{n}(x)=\int_{-\infty}^{x} m_{1}(x-y) u_{n}^{\prime}(y) d y+f \int_{-1}^{x} m_{2}(x-y) p_{n-1}(y) d y, \quad|x|<1,
$$

where the $m_{i}(x), i=1,2$, are approximated to an order of $\alpha$ appropriate to the term in $u_{n}^{\prime}(y), p_{n-1}(y)$ multiplying them. It is $p_{n-1}(y)$ rather than $p_{n}(y)$ that occurs in this equation because of the fact that, for both types of approximation, $m_{2}(x)$ is proportional to $\alpha$. If the problem has been solved to order $n-1$ then $p_{n-1}(y)$ will be known and the second term on the right of (2.38) is known. Since, to order zero the problem reduces to that of [7] and can be solved, it is clearly possible to solve (2.34) provided one can handle equations of such a form with $W(x)$ given by

$$
W(x)=\int_{-\infty}^{x} m_{1}(x-y) u^{\prime}(y) d y+\phi(x),
$$

where $\phi(x)$ is a known function. In this paper we will consider only up to first order so that, given the solution of the problem considered in [7], the only new situation that must be considered is the case where

$$
\phi(x)=f \int_{-1}^{x} m_{2}^{(1)}(x-y) p_{0}(y) d y,
$$

where $m_{2}^{(1)}(x)$ is $m_{2}(x)$ to order $\alpha$ while $p_{0}(y)$ is the limit of $p(y)$ given in [7], but with the contact interval occurring in this more general problem. This means that the dimensionless coordinates in $p_{0}(y)$ will be just those defined by (2.24). Any error introduced by this procedure will be of higher order in $\alpha$, and so may be neglected. The use of any other contact interval might introduce branch points into the region of integration. 
As in $[6,7]$, the non-trivial part of the quantity $W(x)$ will be shown to obey a non-singular integral equation. Let the punch shape be given by a polynomial of degree $m+1$ so that $u^{\prime}(x)$ in the interval $[-1,1]$ is given by

$$
u^{\prime}(x)=d(x)=\sum_{r=0}^{m} d_{r} x^{r}
$$

Let

$$
W(x)=\Delta(x)+q(x)+\phi(x),
$$

where

$$
\Delta(x)=\int_{-\infty}^{-1} m_{1}(x-y)\left(u^{\prime}(y)-d(y)\right) d y
$$

and

$$
\begin{aligned}
q(x) & =\int_{-\infty}^{x} m_{1}(x-y) d(y) d y \\
& =\int_{0}^{\infty} m_{1}(y) d(x-y) d y,
\end{aligned}
$$

which will also be a polynomial and of the same order as $d(x)$. In order to substitute $u^{\prime}(y)$, expressed in terms of $W(y)$, back into (2.43) we must be able to solve the second equation of (2.34) for $u^{\prime}(y)$ in terms of $p(x)$. For this we need a function $l_{1}(x)$ such that (compare with (2.7))

$$
\int_{-\infty}^{\infty} l_{1}(x-y) m_{1}(y-z) d y=\delta(x-z)
$$

Since $k_{1}(x)$ is zero for negative $x$ the lower limit of the integral is $z$. The function may in principle be found by defining (see (2.21), (2.37))

$$
L_{1}(t)=-\frac{1}{2 \pi \eta} \int_{-\infty}^{\infty} \exp (i \omega t) \frac{\Gamma_{2}(\omega / V)}{2 \bar{\mu}(\omega) \Gamma_{1}(\omega / V)} d \omega,
$$

and putting (see (2.26))

$$
l_{1}(x)=\sigma L_{1}(\sigma x) .
$$

In fact $l_{1}(x)$ is also zero for negative $x$. This may be seen by expanding the integrand of (2.46) in terms of the viscoelastic functions and using the Faltung theorem [10]. Noting that one is really dealing with continuous matrix multiplication, this statement corresponds to the fact that the inverse of a triangular matrix is also triangular. So $(2.45)$ becomes

$$
\int_{z}^{x} l_{1}(x-y) m_{1}(y-z) d y=\delta(x-z)
$$


and from (2.34), (2.36)

$$
u^{\prime}(y)=-\frac{\eta}{\pi} \int_{-\infty}^{y} l_{1}(y-x) \int_{-1}^{1} \frac{p(z)}{z-x} d z d x, \quad y<-1 .
$$

Therefore, using the definition of $\Delta(x)$ as given by (2.43), one has

$$
\Delta(x)=-\frac{\eta}{\pi} \int_{-\infty}^{-1} T(x, y) \int_{-1}^{1} \frac{p(z)}{z-y} d z d y-\int_{-\infty}^{-1} m_{1}(x-y) d(y) d y,
$$

where [7]

$$
T(x, y)=\int_{y}^{-1} m_{1}\left(x-x^{\prime}\right) l_{1}\left(x^{\prime}-y\right) d x^{\prime}, \quad|x|<1, \quad y<-1 .
$$

By using a similar argument to that given in [7] one transforms (2.50) into

$$
\Delta(x)=\int_{-1}^{1} K\left(x, x^{\prime}\right) W\left(x^{\prime}\right) d x^{\prime}-\int_{-\infty}^{-1} m_{1}(x-y) d(y) d y,
$$

where

$$
K\left(x, x^{\prime}\right)=-\frac{\sin \pi \theta}{\pi} \int_{-\infty}^{-1} \frac{T(x, y) f\left(x^{\prime}\right)}{\left(x^{\prime}-y\right) g(y)} d y,
$$

the quantities $f(x)$ and $g(y)$ being given by

$$
\begin{aligned}
& f(x)=\left(\frac{1+x}{1-x}\right)^{\theta}, \\
& g(y)=\left(\frac{y+1}{y-1}\right)^{\theta},
\end{aligned}
$$

and

$$
\left.\theta=\frac{1}{\pi} \tan ^{-1} \frac{\eta}{c} \quad \text { (evaluated in }[0, \pi]\right)
$$

Continuing along identical lines to [7] one finally obtains the integral equation for $\Delta(x)$,

$$
\Delta(x)=\int_{-1}^{1} K\left(x, x^{\prime}\right) \Delta\left(x^{\prime}\right) d x^{\prime}+n(x)
$$

where

$$
n(x)=-\int_{-\infty}^{-1} \frac{T(x, y) B(y)}{g(y)} d y+\int_{-1}^{1} K\left(x, x^{\prime}\right) \phi\left(x^{\prime}\right) d x^{\prime},
$$

where $\phi(x)$ is given by (2.40) and $B(y)$ is a polynomial of degree $m$ given by (A.14). Once (2.56) is solved then $\Delta(x)$, and therefore $W(x)$, is known, apart from the dependence on the contact length parameters. Two subsidiary conditions are 
required to determine these, namely the condition that $p(x)$ be bounded [7]:

$$
\frac{\sin \pi \theta}{\pi} \int_{-1}^{1} \frac{W(y) f(y)}{1+y} d y \equiv V_{1}+B(-1)=0
$$

where

$$
\begin{aligned}
& V_{1}=\frac{\sin \pi \theta}{\pi} \int_{-1}^{1} \frac{\Delta(y) f(y)}{1+y} d y+I_{1}, \\
& I_{1}=\frac{\sin \pi \theta}{\pi} \int_{-1}^{1} \frac{\phi(y) f(y)}{1+y} d y,
\end{aligned}
$$

and the definitions of the load per unit length $L$, which is assumed to be specified [7]:

$$
\begin{aligned}
\frac{\eta L}{\pi \theta(b-a)} & =\frac{\sin \pi \theta}{2 \pi \theta} \int_{-1}^{1} f(x) W(x) d x \\
& =V_{2}+\frac{\sin \pi \theta}{2 \pi \theta} \int_{-1}^{1} f(x) q(x) d x,
\end{aligned}
$$

where

$$
\begin{aligned}
V_{2} & =\frac{\sin \pi \theta}{2 \pi \theta} \int_{-1}^{1} \Delta(x) f(x) d x+I_{2}, \\
I_{2} & =\frac{\sin \pi \theta}{2 \pi \theta} \int_{-1}^{1} \phi(x) f(x) d x .
\end{aligned}
$$

The quantities $V_{i}, I_{i}, i=1,2$, will prove convenient later. The relations (2.56), (2.58), (2.61) fully determine the system, allowing the various quantities of interest to be calculated. In particular, the pressure distribution is given by

$$
\begin{aligned}
p(x)=\frac{1}{\sqrt{\left(c^{2}+\eta^{2}\right)}}[\cos \pi \theta & (\Delta(x)+\phi(x))+\frac{B(x)}{f(x)} \\
& \left.+\frac{\sin \pi \theta}{\pi f(x)}(\mathrm{P}) \int_{-1}^{1} \frac{f(y)(\Delta(y)+\phi(y))}{y-x}\right] d y,
\end{aligned}
$$

which follows by solving (2.34) (see [11, 4]). Clearly this is bounded by virtue of (2.58). As in the non-inertial case with friction, $p( \pm 1)$ are not in general zero in the presence of viscoelasticity. The coefficient of friction due to deformation, $f_{D}$, is given by

$$
f_{D}=-\int_{-1}^{1} R(y) f(y) W(y) d y / \int_{-1}^{1} f(y) W(y) d y
$$

where $R(y)$ is a polynomial defined in [7]. It is essentially $B(y)$ with $d(x)$ replacing $q(x)$ and $(-\theta)$ replacing $\theta$. 


\section{Small velocity approximation}

The quantities $m_{i}(x), i=1,2$, will first be expanded in power series in $V$. It will be assumed that $\beta$ defined by (2.32) is a constant. One can show from (2.19), (2.20) that

$$
\frac{2 \bar{\mu}(\omega) \Gamma_{1}(\omega / V)}{\Gamma_{2}(\omega / V)}=a_{0} \bar{\mu}(\omega)+a_{1}+\frac{a_{2}}{\bar{\mu}(\omega)}+\ldots
$$

where

and

$$
\begin{aligned}
& a_{0}=2(\beta-1), \\
& a_{1}=\frac{1}{2} \rho\left(3 \beta^{2}-4 \beta+3\right) V^{2}, \\
& a_{2}=\frac{1}{4} \rho^{2}\left(5 \beta^{3}-6 \beta^{2}+2 \beta+1\right) V^{4},
\end{aligned}
$$

$$
\frac{\Gamma_{3}(\omega / V)}{\Gamma_{2}(\omega / V)}=b_{0}+\frac{b_{1}}{\bar{\mu}(\omega)}+\frac{b_{2}}{(\bar{\mu}(\omega))^{2}}+\ldots
$$

where

$$
\begin{aligned}
& b_{0}=-\beta, \\
& b_{1}=-\frac{1}{4} \rho\left(3 \beta^{2}-2 \beta+1\right) V^{2}, \\
& b_{2}=-\frac{1}{8} \rho^{2}\left(4 \beta^{3}-3 \beta^{2}+1\right) V^{4} .
\end{aligned}
$$

Only terms up to order $V^{2}$ will be considered from here on. Taking inverse FTs and recalling (2.28), (2.31), (2.37) one has

$$
\begin{aligned}
& m_{1}(x)=\mu_{T}(x)-\eta a_{1} \delta(x), \\
& m_{2}(x)=\eta b_{1} \gamma_{T}(x),
\end{aligned}
$$

to order $V^{2} / c_{T}^{2}$. The second relation allows us to evaluate $\phi(x)$ defined by (2.40). Note that it is of order $V^{2} / c_{T}^{2}$. From the first relation and (2.48) we can deduce $l_{1}(x)$. In fact, it is better to work with FT quantities initially. From (2.48)

$$
l_{1}(\omega) \bar{m}_{1}(\omega)=\bar{l}_{1}(\omega)\left(\bar{\mu}_{T}(\omega)-\eta a_{1}\right)=1,
$$

so that, to order $\delta_{T}$,

$$
\bar{l}_{1}(\omega)=\bar{\gamma}_{T}(\omega)+\eta a_{1} \bar{\gamma}^{2}(\omega)
$$

Inverting the FT gives

$$
l_{1}(x)=\gamma_{T}(x)+\eta a_{1} \int_{0}^{x} \gamma_{T}(x-y) \gamma_{T}(y) d y,
$$

and $T(x, y)$ defined by $(2.51)$ has the form

$$
T(x, y)=T_{0}(x, y)+\eta a_{1} \int_{\nu}^{-1} \mu_{T}\left(x-x^{\prime}\right) \int_{0}^{x^{\prime}-y} \gamma_{T}\left(x^{\prime}-y-z\right) \gamma_{T}(z) d z d x^{\prime},
$$


where $T_{0}(x, y)$ is the non-inertial limit given by $[6,7]$ :

$$
T_{0}(x, y)=\int_{y}^{-1} \mu_{T}\left(x-x^{\prime}\right) \gamma_{T}\left(x^{\prime}-y\right) d x^{\prime} .
$$

These relations allow us to write down (2.56), (2.58), (2.60) more explicitly and consider their solution. This will be done in the next section under the assumption that the viscoelastic functions are characterized by a discrete spectrum.

It is clear from (3.1)-(3.4) that this type of expansion will be valid provided the quantity $\rho V^{2} / \bar{\mu}(\omega)$ is fairly small. The quantity $\bar{\mu}(\omega)$ is generally smallest at the low frequency (large time) limit so that a convenient criterion of the validity of the velocity expansion is that $\rho V^{2} / \bar{\mu}(0)$ be small.

\section{Discrete spectrum}

While the method described in the last section requires no special assumption on the nature of the viscoelastic behaviour it is convenient to work it out in detail for the standard discrete spectrum model. We let $\mu_{T}(x), \gamma_{T}(x)$ have the form $[6,7]$

$$
\begin{aligned}
& \mu_{T}(x)=g_{0} \delta(x)+\theta(x) \sum_{i=1}^{N} g_{i} \exp \left(-\alpha_{i} x\right), \\
& \gamma_{T}(x)=h_{0} \delta(x)+\theta(x) \sum_{i=1}^{N} h_{i} \exp \left(-\beta_{i} x\right),
\end{aligned}
$$

where $\theta(x)$ is the unit step-function, zero for negative $x$, while the $\alpha_{i}, \beta_{i}$ are related to the time constants for creep and relaxation as specified in $[6,7]$. Also certain relations exist between these and the constants $g_{i}, h_{i}$ which are consequences of (2.5). These are explicitly given in [6]. The quantity $T_{0}(x, y)$ of $(3.10)$ has the form $[6,7]$

$$
T_{0}(x, y)=\sum_{i, j=1}^{N} \frac{g_{i} h_{j}}{\alpha_{i}-\beta_{j}} F_{i j}(x, y)
$$

where

$$
F_{i j}(x, y)=\exp \left(-\alpha_{i}(x+1)+\beta_{j}(y+1)\right),
$$

while the second term on the right of (3.9), which we denote by $T_{1}(x, y)$, can be shown to be given by

$$
T_{1}(x, y)=\sum_{i, j=1}^{N} g_{i}\left(T_{i j}^{(0)}+(y+1) T_{i j}^{(1)}\right) F_{i j}(x, y),
$$


where

$$
\begin{aligned}
T_{i j}^{(0)} & =\frac{\eta a_{1} h_{j}}{\alpha_{i}-\beta_{j}}\left(2 h_{0}-\frac{h_{j}}{\alpha_{i}-\beta_{j}}+2 \sum_{k=1, k \neq j}^{N} \frac{h_{k}}{\beta_{k}-\beta_{j}}\right), \\
T_{i j}^{(1)} & =\frac{-\eta a_{1} h_{j}^{2}}{\alpha_{i}-\beta_{j}} .
\end{aligned}
$$

The relationships between the $\left\{g_{i}, \alpha_{j}\right\}$ and the $\left\{h_{i}, \beta_{j}\right\}$ mentioned above have been used in the derivation of (4.2). One finally has

$$
T(x, y)=\sum_{i, j=1}^{N} g_{i}\left(t_{i j}^{(0)}+t_{i j}^{(1)} \frac{\partial}{\partial \beta_{j}}\right) F_{i j}(x, y)
$$

where

$$
\begin{aligned}
& t_{i j}^{(0)}=\frac{h_{j}}{\alpha_{i}-\beta_{j}}+T_{i j}^{(0)}, \\
& t_{i j}^{(1)}=T_{i j}^{(1)} .
\end{aligned}
$$

The quantity $\Delta(x)$ of $(2.43)$ has the form

$$
\Delta(x)=\sum_{k=1}^{N} g_{k} C_{k} \exp \left(-\alpha_{i} x\right)
$$

as in [7], where

$$
C_{k}=\int_{-\infty}^{-1} \exp \left(\alpha_{k} y\right)\left(u^{\prime}(y)-d(y)\right) d y,
$$

since the delta function in $m_{1}(x)$ given by (3.5) does not contribute for $|x|<1$. The polynomial $q(x)$ has the form

$$
q(x)=\left(g_{0}-\eta a_{1}\right) d(x)+\sum_{i=1}^{N} g_{i} d_{i}(x)
$$

where [6]

$$
d_{i}(x)=\sum_{k=0}^{m}(-1)^{k} \frac{d^{(k)}(x)}{\alpha_{i}^{k+1}}
$$

the bracketed superscript indicating differentiation.

It follows from (2.53) that

$$
K\left(x, x^{\prime}\right)=\frac{\sin \pi \theta}{\pi} \sum_{i, j=1}^{N} g_{i}\left(t_{i j}^{(0)}+t_{i j}^{(1)} \frac{\partial}{\partial \beta_{j}}\right)\left(E\left(x^{\prime}, \beta_{j}\right) \exp \left(\beta_{j}-\alpha_{i}(x+1)\right)\right)
$$

where

$$
E\left(x^{\prime}, \beta_{j}\right)=\int_{-\infty}^{-1} \frac{\exp \left(\beta_{j} y\right) f\left(x^{\prime}\right)}{\left(y-x^{\prime}\right) g(y)} d y
$$


an integral which is evaluated in Appendix 1 of [7] to be

$$
E\left(x^{\prime}, \beta_{j}\right)=-\beta_{j} \exp \left(\beta_{j} x\right) \int_{x^{\prime}}^{1} \exp \left(-\beta_{j} y\right) \frac{\left(A_{j}(y+1)+B_{j}\right)}{(y+1)} f(y) d y+A_{j} f\left(x^{\prime}\right)
$$

where

$$
\begin{aligned}
& A_{j}=-\frac{\Gamma(1-\theta)}{\sqrt{\left(2 \beta_{j}\right)}} W_{\theta-i, 0}\left(2 \beta_{j}\right), \\
& B_{j}=\frac{\Gamma(1-\theta)}{\beta_{j}} W_{\theta, \mathrm{l}}\left(2 \beta_{j}\right),
\end{aligned}
$$

the functions $W_{\theta-\frac{1}{2}, 0}\left(2 \beta_{j}\right)$ and so on being confluent hypergeometric functions. Note that $K\left(x, x^{\prime}\right)$ given by (4.12) is of the standard separable form, that is consists of a sum of products of functions $k_{i}^{(1)}, k_{i}^{(2)}$ of $x, x^{\prime}$ individually. When the kernel has this property a linear integral equation may be transformed into algebraic form provided that the inhomogeneous term is expressible as a sum of the $k_{i}^{(1)}$. This is assured in our context if $T(x, y)$ has the separable form. By following a line of argument essentially identical to that in [7], one can show that (2.56) becomes

$$
\begin{aligned}
\sum_{j=1}^{N}\left\{\left(t_{i j}^{(0)}+\right.\right. & \left.\left.t_{i j}^{(1)}\left(\frac{\partial}{\partial \beta_{j}}+1\right)\right) N_{j}^{(1)}-\sum_{k=1}^{N} g_{k} C_{k}\left(T_{i j}^{(0)}+\frac{t_{i j}^{(1)}}{\alpha_{k}-\beta_{j}}\right) \frac{\exp \left(\alpha_{k}-\beta_{j}\right)}{\alpha_{k i}-\beta_{j}}\right\} \\
= & \sum_{j=1}^{N}\left(t_{i j}^{(0)}+t_{i j}^{(1)}\left(\frac{\partial}{\partial \beta_{j}}+1\right)\right) N_{j}^{(2)},
\end{aligned}
$$

where

$$
\begin{aligned}
N_{j}^{(1)} & =\sum_{k=1}^{N} \frac{g_{k} C_{k} S\left(\beta_{j}, \alpha_{k}\right)}{\alpha_{k}-\beta_{j}}, \\
S(\beta, \alpha) & =\Gamma(1-\theta)\left\{\frac{1}{\sqrt{(2 \alpha)}} W_{\theta, \mathrm{j}}(2 \beta) M_{\theta-\mathrm{t}, 0}(2 \alpha)-\frac{\theta}{\sqrt{(2 \beta)}} W_{\theta-\mathrm{t}, 0}(2 \beta) M_{\theta, \mathrm{j}}(2 \alpha)\right\},
\end{aligned}
$$

the functions $M_{\theta, 3}(2 \alpha)$ and so on being the other confluent hypergeometric functions and

$$
N_{j}^{(2)}=\int_{-\infty}^{-1} \frac{\exp \left(\beta_{j} y\right) B(y)}{g(y)} d y-\frac{\sin \pi \theta}{\pi} \int_{-1}^{1} E\left(x, \beta_{j}\right) \phi(x) d x,
$$

where $E\left(x, \beta_{j}\right)$ is given by (4.14). The relation (4.16) is not as simple as the corresponding one in [7] because the second term on the left prevents cancellation of the factor operating on $N_{j}^{(1)}, N_{j}^{(2)}$. From a relation given in Appendix 1 of [7] one can show that

$$
\frac{\partial}{\partial \beta} S(\beta, \alpha)=-S(\beta, \alpha)+S_{1}(\beta, \alpha)
$$


where

$$
S_{1}(\beta, \alpha)=\Gamma(1-\theta) \theta\left[\frac{1}{\sqrt{ }(\alpha \beta)} W_{\theta-\mathbf{i}, 0}(2 \beta) M_{\theta-\mathbf{k}, 0}(2 \alpha)-\frac{(\theta-1)}{\beta} W_{\theta-1,-\frac{1}{\mathbf{b}}}(2 \beta) M_{\theta, \mathbf{z}}(2 \alpha)\right]
$$

so that (4.16) becomes

$$
\begin{aligned}
\sum_{k, j=1}^{N} \frac{g_{k} C_{k}}{\alpha_{k}-\beta_{j}}\left\{\left(t_{i j}^{(0)}\right.\right. & \left.+t_{i j}^{(1)}\left(1+\frac{1}{\alpha_{k}-\beta_{j}}\right)\right) S\left(\beta_{j}, \alpha_{k}\right) \\
& \left.+t_{i j}^{(1)} S_{1}\left(\beta_{j}, \alpha_{k}\right)-\left(T_{i j}^{(0)}+\frac{t_{i j}^{(1)}}{\alpha_{k}-\beta_{j}}\right) \exp \left(\alpha_{k}-\beta_{j}\right)\right\} \\
= & \sum_{j=1}^{N}\left(t_{i j}^{(0)} N_{j}^{(2)}+t_{i j}^{(1)} N_{j}^{(21)}\right),
\end{aligned}
$$

where

$$
N_{j}^{(21)}=\int_{-\infty}^{-1} \frac{\exp \left(\beta_{j} y\right) B(y)(y+1)}{g(y)} d y .
$$

Note that the fact that $\phi(x)$ is a first order quantity has been used in writing (4.22). Also $t_{i j}^{(0)}$ may be replaced by its zeroth order term when multiplying the term containing $\phi(x)$ in $N_{j}^{(2)}$. One may achieve slight simplication of these equations by decomposing the $C_{i}$ into a non-inertial part and a first order inertial correction.

It remains to express the last term on the left of (4.21) more explicitly. This involves evaluation of $\phi(x)$ defined by (2.40) and given here by

$$
\phi(x)=\eta f b_{1}\left(h_{0} p_{0}(x)+\sum_{i=1}^{N} h_{i} \exp \left(-\beta_{i} x\right) \int_{-1}^{x} \exp \left(\beta_{i} y\right) p_{0}(y) d y\right) .
$$

The quantity $p_{0}(x)$ may be deduced from results in [7] to be:

$$
\begin{aligned}
p_{0}(x)=\frac{1}{\sqrt{\left(c^{2}+\eta^{2}\right)}}\left[\frac{B(x)-B(-1)}{f(x)}+\sin \pi \theta \sum_{i=1}^{N} C_{i} g_{i}\right. \\
\left.\quad\left(\alpha_{i} \exp \left(-\alpha_{i} x\right) \int_{-1}^{x} \frac{\exp \left(\alpha_{i} y\right)\left(F_{i}(y-1)+G_{i}\right)}{(1-y) f(y)} d y\right)\right],
\end{aligned}
$$

where $B(x)$ is given by (A.14) and where the $C_{i}$ may be replaced by those obtained when inertial effects are neglected, while

$$
\begin{aligned}
F_{i} & =\frac{M_{\theta-t, 0}\left(2 \alpha_{i}\right)}{\sin \pi \theta \sqrt{\left(2 \alpha_{i}\right)}}, \\
G_{i} & =\frac{\theta M_{\theta, \mathbf{t}}\left(2 \alpha_{i}\right)}{\alpha_{i} \sin \pi \theta} .
\end{aligned}
$$

It is not possible to evaluate the integrals occurring in these expressions explicitly. 
However, $\phi(x)$ can be evaluated numerically from (4.23), (4.24) as can $E\left(x, \beta_{j}\right)$ given by (4.14), allowing numerical evaluation of the second integral in (4.18).

The equations (4.21) together with the two conditions (2.58), (2.61) constitute a system of equations for the $C_{i}$ and the contact length parameters $a, b$. These can in general only be solved numerically.

More explicitly $V_{1}, V_{2}$ of (2.59), (2.62) are given by

$$
\begin{aligned}
& V_{1}=\sum_{i=1}^{N} \frac{C_{i} g_{i}}{\sqrt{\left(2 \alpha_{i}\right)}} M_{\theta-t, 0}\left(2 \alpha_{i}\right)+I_{1}, \\
& V_{2}=\sum_{i=1}^{N} \frac{C_{i} g_{i}}{2 \alpha_{i}} M_{\theta, \frac{1}{2}}\left(2 \alpha_{i}\right)+I_{2} .
\end{aligned}
$$

The quantities $I_{1}, I_{2}$ can be evaluated numerically. They, along with the extra terms in (4.21), constitute the inertial corrections to the results of [7]. Once the $C_{i}$ and $a, b$ are known, the quantity $f_{D}$ of (2.65) may be evaluated as can the pressure given by (2.64).

\section{Cylindrical punch}

This section deals with the case where the punch shape is cylindrical or parabolic. The steps and results are closely analogous in many respects to those of the corresponding section in [7]. Initially, no assumption will be made about viscoelastic behaviour. Ultimately, however, the discrete spectrum model will be used for illustration. This punch shape corresponds to $m=1$ in (2.41) so that

$$
u^{\prime}(x)=d(x)=d_{0}+d_{1} x, \quad|x|<1,
$$

where, in terms of $R$, the cylinder radius

$$
\begin{aligned}
& d_{0}=\frac{b+a}{2 R}, \\
& d_{1}=\frac{b-a}{2 R} .
\end{aligned}
$$

The polynomial $q(x)$ of (2.44) has the form

where

$$
q(x)=q_{0}+q_{1} x
$$

$$
\begin{aligned}
& q_{0}=d_{0}\left(\Sigma_{0}-\eta a_{1}\right)-(V / R) \Sigma_{1}, \\
& q_{1}=d_{1}\left(\Sigma_{0}-\eta a_{1}\right),
\end{aligned}
$$


the quantities $\Sigma_{0}, \Sigma_{1}$ being given by

$$
\begin{aligned}
& \Sigma_{0}=\int_{0}^{\infty} \mu_{T}(y) d y=G_{1}(\infty), \\
& \Sigma_{1}=\sigma \int_{0}^{\infty} \mu_{T}(y) y d y=\int_{0}^{\infty}\left(G_{1}(\infty)-G_{1}(t)\right) d t,
\end{aligned}
$$

where $\sigma$ is defined by (2.27) while $G_{1}(t)$ is the relaxation function for shear, closely related to $\mu(t)$ [6]. The polynomial $B(y)$ of (A.14) has the form

$$
B(y)=q_{0}-k q_{1}+q_{1}(y+1),
$$

where

$$
k=1-2 \theta \text {. }
$$

The subsidiary conditions (2.58), (2.61) become in this case

and

$$
V_{1}=-q_{0}+q_{1} k
$$

$$
\frac{\eta L}{2 R d_{1} \pi \theta}=V_{2}+q_{0}+q_{1} \theta
$$

The relation (5.9) may be written as

$$
g_{0} \frac{d_{10}^{2}(1-\theta)}{d_{1}}=V_{2}+q_{0}+q_{1} \theta
$$

where $d_{10}$ is the quantity $d_{1}$ in the limit of zero viscoelasticity and neglect of inertial effects, defined by

$$
d_{10}^{2}=\frac{\eta L}{2 g_{0} \pi R \theta(1-\theta)},
$$

while $g_{0}$ is the short-time limit of the shear modulus.

Little further can be said about (2.56) without specific assumptions about viscoelastic behaviour. The expression (2.65) for $f_{D}$ becomes [7]

$$
f_{D}=-d_{0}-d_{1} k+\frac{1}{g_{0}}\left(\frac{d_{1}}{d_{10}}\right)^{2}\left(V_{3}+q_{0}-\frac{1}{3} q_{1} k\right)
$$

where

$$
\begin{aligned}
& V_{3}=\frac{\sin \pi \theta}{2 \pi \theta(1-\theta)} \int_{-1}^{1} f(y) \Delta(y)(1-y) d y+I_{3}, \\
& I_{3}=\frac{\sin \pi \theta}{2 \pi \theta(1-\theta)} \int_{-1}^{1} f(y) \phi(y)(1-y) d y .
\end{aligned}
$$


In the case of a discrete spectrum (5.8) and (5.9) become, by virtue of (4.26),

$$
\begin{gathered}
\sum_{i=1}^{N} \frac{C_{i} g_{i}}{\sqrt{\left(2 \alpha_{i}\right)}} M_{\theta-\downarrow, 0}\left(2 \alpha_{i}\right)=-q_{0}+q_{1} k-I_{1}, \\
\frac{g_{0} d_{10}^{2}(1-\theta)}{d_{1}}=\sum_{i=1}^{N} \frac{C_{i} g_{i}}{2 \alpha_{i}} M_{\theta, \mathbf{4}}\left(2 \alpha_{i}\right)+I_{2}+q_{0}+q_{1} \theta,
\end{gathered}
$$

while (2.56) takes the form (4.21) with

$$
\begin{aligned}
& N_{j}^{(2)}=\frac{\Gamma(1-\theta)}{\beta_{j}}\left[\left(q_{0}-k q_{1}\right) W_{\theta, \mathrm{t}}\left(2 \beta_{j}\right)-q_{1}\left(\frac{2}{\beta_{j}}\right)^{t}(1-\theta) W_{\theta-\mathrm{t}, 1}\left(2 \beta_{j}\right)\right] \\
& -\frac{\sin \pi \theta}{\pi} \int_{-1}^{1} E\left(x, \beta_{j}\right) \phi(x) d x, \\
& N_{j}^{(21)}=\left(\frac{2}{\beta_{j}^{3}}\right)^{\frac{1}{t}} \Gamma(2-\sigma)\left[\left(\frac{2}{\beta}\right)^{\frac{1}{2}} q_{1}(2-\theta) W_{\theta-1 \xi},\left(2 \beta_{j}\right)-\left(q_{0}-k q_{1}\right) W_{\theta-1,1}\left(2 \beta_{j}\right)\right],
\end{aligned}
$$

where $E\left(x, \beta_{j}\right)$ is defined by (4.14). These relationships may then be solved numerically for the $C_{i}, a, b$, so that $f_{D}$ given by (5.12) with

$$
V_{3}=\sum_{i=1}^{N} \frac{C_{i} g_{i}}{\left(2 \alpha_{i}\right)^{\frac{1}{3}}} M_{\theta-\mathrm{i}, 1}\left(2 \alpha_{i}\right)+I_{3}
$$

may be calculated, as also may $p(x)$ given by (2.64), though the latter task is rendered more subtle by the singular kernel and the fact that the terms involving $\phi(x)$ have to be handled numerically. Building in the boundary condition (5.15) gets rid of explicit singularities apart from that occurring in the kernel.

Note that $p_{0}(x)$ given by (4.24) becomes with the help of (5.6)

$$
\begin{aligned}
p_{0}(x)=\frac{1}{\sqrt{\left(c^{2}+\eta^{2}\right)}}\left[q_{1}(1+x)^{1-\theta}(1-x)^{\theta}+\sin \pi \theta \sum_{i=1}^{N} C_{i} g_{i}\right. \\
\left.\quad \times\left(\alpha_{i} \exp \left(-\alpha_{i} x\right) \int_{-1}^{x} \frac{\exp \left(\alpha_{i} y\right)\left(F_{i}(y-1)+G_{i}\right)}{(1-y) f(y)} d y\right)\right] .
\end{aligned}
$$

Also $\Sigma_{0}, \Sigma_{1}$ defined by (5.5) become in the discrete spectrum case

$$
\begin{aligned}
& \Sigma_{0}=\sum_{i=1}^{N} \frac{g_{i}}{\alpha_{i}}, \\
& \Sigma_{1}=\sigma \sum_{i=1}^{N} \frac{g_{i}}{\alpha_{i}^{2}} .
\end{aligned}
$$

Equations (4.16), (5.15), (5.10) may be solved numerically for the $C_{i}, a, b$ without any difficulties in principle. However, the details are extremely complex, far more so than in the non-inertial case. It would be somewhat easier though less 
interesting to solve an inverse problem as was done in [7]. However, the details here are again far more complex and involve nothing new. By making a different kind of approximation much simpler solutions will be derived in the next section which allow parameters of interest to be evaluated numerically with considerably less effort.

The results of this section will not find much application to the building of general models of frictional processes because of their complexity and of course because of the idealized nature of the problem. It is more in specific engineering situations where the input parameters are largely specified that they may be of interest.

It is possible that the formalism described in Section 3 might prove simpler for a parametrization of viscoelastic behaviour different to (4.1). This point will not be examined further here.

\section{Small viscoelasticity approximation}

The second type of approximation considered is to let $\mu(t), \lambda(t)$ have the form

$$
\begin{gathered}
\mu(t)=g_{0}\left(\delta(t)+\varepsilon_{1}(t)\right), \\
\lambda(t)=l_{0}\left(\delta(t)+\varepsilon_{2}(t)\right),
\end{gathered}
$$

where $\varepsilon_{1}(t), \varepsilon_{2}(t)$ are assumed small. Then it follows that

$$
\begin{aligned}
& \bar{\mu}(\omega)=g_{0}\left(1+\bar{\varepsilon}_{1}(\omega)\right), \\
& \bar{\lambda}(\omega)=l_{0}\left(1+\bar{\varepsilon}_{2}(\omega)\right) .
\end{aligned}
$$

Recalling (2.21) we have

$$
\begin{aligned}
R_{1}(\omega) & =\frac{2 \bar{\mu}(\omega) \Gamma_{1}(\omega / V)}{\Gamma_{2}(\omega / V)} \\
& =D_{10}+\sum_{i=1}^{2} D_{1 i} \bar{\varepsilon}_{i}(\omega),
\end{aligned}
$$

where

$$
\begin{aligned}
& D_{10}=\left.R_{1}(\omega)\right|_{\bar{\varepsilon}_{1}=\bar{\varepsilon}_{2}=0}, \\
& D_{1 i}=\left.\frac{\partial}{\partial \bar{\varepsilon}_{i}} R_{1}(\omega)\right|_{\bar{\varepsilon}_{1}=\bar{\varepsilon}_{2}=0} .
\end{aligned}
$$

These coefficients are independent of $\omega$. More explicitly, they have the following form (see (2.19)):

$$
D_{10}=\frac{4 g_{0}\left(A_{03}^{2}-A_{01} A_{02}\right)}{\delta_{0 T} A_{01}},
$$


where (see (2.13), (2.16))

$$
\begin{aligned}
& \delta_{0 T}=\frac{\rho V^{2}}{g_{0}}, \\
& \delta_{0 L}=\frac{\rho V^{2}}{l_{0}+2 g_{0}},
\end{aligned}
$$

and (see (2.20))

Also

$$
\begin{aligned}
& A_{01}=\left(1-\delta_{0 L}\right)^{\frac{1}{2}}, \\
& A_{02}=\left(1-\delta_{0 T}\right)^{\frac{1}{2}}, \\
& A_{03}=1-\frac{1}{2} \delta_{0 T} .
\end{aligned}
$$

$$
D_{12}=-2 \kappa l_{0} \frac{A_{03}^{2}}{A_{01}^{3}}
$$

where

$$
\kappa=\frac{g_{0}^{2}}{\left(l_{0}+2 g_{0}\right)^{2}}
$$

Similarly,

$$
\begin{aligned}
R_{2}(\omega) & =\frac{\Gamma_{3}(\omega / V)}{\Gamma_{2}(\omega / V)} \\
& =D_{20}+\sum_{i=1}^{2} D_{2 i} \bar{\varepsilon}_{i}(\omega),
\end{aligned}
$$

where

$$
\begin{aligned}
D_{20} & =\left.R_{2}(\omega)\right|_{\bar{\varepsilon}_{1}=\bar{\varepsilon}_{2}=0}=\frac{A_{01} A_{02}-A_{03}}{\frac{1}{2} \delta_{0 T} A_{01}}, \\
D_{21} & =\left.\frac{\partial}{\partial \bar{\varepsilon}_{1}} R_{2}(\omega)\right|_{\bar{e}_{1}=\bar{e}_{2}=0} \\
& =D_{20}+\frac{1}{A_{02}}-\frac{1}{A_{01}}+2 \kappa \frac{A_{03}}{A_{01}^{3}}, \\
D_{22} & =\left.\frac{\partial}{\partial \bar{\varepsilon}_{2}} R_{2}(\omega)\right|_{\bar{e}_{1}=\bar{\varepsilon}_{2}=0} \\
& =\frac{l_{0} \kappa}{g_{0}} \frac{A_{03}}{A_{01}^{s}} .
\end{aligned}
$$


Therefore we write $k_{i}(x), i=1,2$, of $(2.26)$ in the form

where

$$
k_{\imath}(x)=D_{i 0}\left(\delta(x)+r_{i}(x)\right) \text {, }
$$

$$
r_{i}(x)=\frac{\sigma}{D_{i 0}} \sum_{j=1}^{2} D_{i j} \varepsilon_{j}(\sigma x),
$$

the parameter $\sigma$ being given by (2.27). It is straightforward if lengthy to check from (6.14) that in the limit of zero viscoelasticity (2.30) reduces to the equation given by Galin [4] in the form expressed in terms of Lamé constants, though not in the form in terms of Young's modulus and Poisson's ratio, due to an error of sign in his equation (9.45). Clearly also from (6.14), $k_{2}(x)$ has the form of (2.31) and the analysis subsequent to that equation goes through. In particular one has (2.34) but with $c$ replaced by

$$
c_{1}=-f D_{20},
$$

and $\eta$ replaced by unity since that parameter was introduced in order to make the treatment of the small velocity approximation correspond closely to the results of [7]; it would serve no purpose here. Therefore one has the correspondence

$$
\begin{aligned}
& m_{1}(x)=-k_{1}(x), \\
& m_{2}(x)=D_{20} r_{2}(x) .
\end{aligned}
$$

The quantity $l_{1}(x)$ of (2.48) has the form

$$
l_{1}(x)=-\frac{1}{D_{10}}\left(\delta(x)-r_{1}(x)\right)
$$

so that $T(x, y)$ of $(2.51)$ has the form

$$
T(x, y)=r_{1}(x-y),
$$

which is first order in viscoelasticity. Therefore the first term on the right of (2.56) may be neglected. Also, from (6.17), (2.40)

$$
\phi(x)=f D_{20} \int_{1}^{x} r_{2}(x-y) p_{0}(y) d y,
$$

where $p_{0}(y)$ is now the pressure distribution in the elastic limit which may be seen, for example from (4.23), to be

$$
p_{0}(x)=\frac{1}{\sqrt{\left(c_{1}^{2}+1\right)}}\left(\frac{B_{0}(x)-B_{0}(-1)}{f(x)}\right)
$$

where $B_{0}(x)$ is given by (A.14) but with $q(x)$ replaced in (A.1) by

$$
q_{0}(x)=-D_{10} d(x) \text {. }
$$

The quantity $\theta$ is given by (2.55) but with $c_{1}$ replacing $c / \eta$. 
Clearly $\phi(x)$ is first order in viscoelasticity so that the second term on the right of (2.57) may also be neglected. One therefore has from (2.56)

$$
\Delta(x)=-\int_{-\infty}^{-1} \frac{r_{1}(x-y) B_{0}(y)}{g(y)} d y .
$$

This explicit expression may be substituted into (2.58), (2.61) which may be solved to first order for $a, b$ thus completing the solution. Formally the equations and results are precisely analogous to the small viscoelasticity results of [7] and so need not be repeated in detail here. The only difference of any consequence is that $V_{i}, i=1,2,3$, of (2.59), (2.62) and (5.13) have the extra terms $I_{i}, i=1,2,3$. Only the expression for $f_{D}$ in the case of a cylindrical punch will be quoted here:

$$
f_{D}=-\frac{4}{3} d_{1 e} k+\frac{1}{\chi_{0}}\left[V_{3}-\frac{V}{R} \chi_{1}\right]
$$

where (see (5.5), (5.11))

$$
\begin{aligned}
& \chi_{0}=-\int_{0}^{\infty} k_{1}(x) d x, \\
& \chi_{1}=-\sigma \int_{0}^{\infty} k_{1}(x) x d x, \\
& d_{1 e}^{2}=\frac{L}{2 \chi_{0} \pi R \theta(1-\theta)},
\end{aligned}
$$

while $V_{3}$ is given by (5.13) with

$$
\Delta(x)=-\chi_{0} d_{1 e} \int_{-\infty}^{-1} r_{1}(x-y)(y-1)^{\theta}(y+1)^{1-\theta} d y,
$$

and $\phi$ is given by (6.20). Note that $\left(-D_{10}\right)$ has been replaced by $\chi_{0}$ in (6.28). This results only in second order errors. A similar substitution is also made below. In the case of a discrete spectrum

$$
r_{i}(x)=\sum_{j=1}^{N} r_{i j} \exp \left(-\alpha_{j} x\right), \quad i=1,2 .
$$

The summation is presumed to include both the time constants for shear and bulk if these are different. For this case $V_{3}$ in (6.24) becomes

$$
V_{3}=\chi_{0} d_{1} \sum_{j=1}^{N}\left(\frac{\mu(2-\theta) r_{i j}}{2 \alpha_{j}^{3}} W_{\theta-i, 1}\left(2 \alpha_{j}\right) M_{\theta-i, 1}\left(2 \alpha_{j}\right)+\frac{f D_{20} r_{2 j}}{\alpha_{j}} \Omega\left(\alpha_{j}, \theta\right)\right)
$$

where

$$
\Omega(\alpha, \theta)=\frac{\alpha \sin ^{2} \pi \theta}{2 \theta(1-\theta) \pi} \int_{-1}^{1} f(y)(1-y) \exp (-\alpha y) \int_{-1}^{\nu} \frac{\exp (\alpha x)(x+1)}{f(x)} d x d y .
$$


In writing (6.30) the relations

$$
\begin{gathered}
\sin \pi \theta=\frac{1}{\sqrt{\left(c_{1}^{2}+1\right)}}, \\
p_{0}(x)=\frac{\chi_{0} d_{1 e}(x+1)}{\sqrt{\left(c_{1}^{2}+1\right) f(x)}}
\end{gathered}
$$

have been used. The quantity $\chi_{1}$ in (6.26) has the form

$$
\chi_{1}=\chi_{0} \sigma \sum_{i=1}^{N} \frac{r_{1 i}}{\alpha_{i}^{2}}
$$

to first order. Therefore the viscoelastic part of $f_{D}$ is characterized completely by the two functions

$$
\Lambda(\alpha, \theta)=\frac{1}{\alpha}\left(1-\frac{\Gamma(2-\theta) W_{\theta-\frac{1}{2}, 1}(2 \alpha) M_{\theta-\underline{t}, 1}(2 \alpha)}{2 \alpha}\right),
$$

which was already isolated in [7], and $\Omega(\alpha, \theta)$ defined by (6.31).

Explicitly, the expression for $f_{D}$ in the case of a discrete spectrum is

$$
f_{D}=-\frac{4}{3} d_{1 e} k+d_{1 e} \sum_{j=1}^{N} \frac{1}{\alpha_{j}}\left(f D_{20} r_{2 j} \Omega\left(\alpha_{j}, \theta\right)-r_{1 j} \Lambda\left(\alpha_{j}, \theta\right)\right) .
$$

It may be shown with the help of (6.5)-(6.15) that in the non-inertial limit, when $\varepsilon_{1}(x)$ and $\varepsilon_{2}(x)$ are equal, (6.36) reduces to the corresponding result in [7]. Note that unless $\varepsilon_{1}(x), \varepsilon_{2}(x)$ are equal then, even in the non-inertial limit, the quantities $r_{2 j}$ are not zero so that the first term of the summation in (6.36) still contributes. Equivalently, the last term in (2.30) is an integral rather than simply proportional to pressure. This integral was the origin of much of the extra complexity in the present paper.

The summation term in (6.36) does not however contain only hysteresis contributions. This may be deduced from the fact that

$$
\lim _{\alpha \rightarrow \infty} \Omega(\alpha, \theta)=\frac{2}{3} \frac{\sin ^{2} \pi \theta}{\pi \theta(1-\theta)},
$$

and the observation that the large $\alpha$ limit corresponds to the zero velocity limit. This implies that in the zero velocity limit the summation term in (6.36) does not vanish as one would expect for the hysteretic contribution. One can, however, reorganize the expression in such a way that the summation term does vanish at large $\alpha$. This can be done by means of a redefinition of $\theta$, replacing that based on (6.16), (6.32). Effectively this amounts to adding a term proportional to $p(x)$ to both sides of (2.34). Specifically, let $D_{20}$ be replaced by

$$
D_{L}=\int_{0}^{\infty} k_{2}(x) d x
$$


which is the large time limit of the combination of derivatives of viscoelastic functions contained in $k_{2}(x)$. Let

$$
\theta_{1}=\frac{1}{\pi} \tan ^{-1}-\frac{1}{-f D_{L}}
$$

One must replace $\phi(x)$ in the analysis by

$$
\phi_{1}(x)=\phi(x)-f D_{20} p_{0}(y) \int_{0}^{\infty} r_{2}(x) d x .
$$

The final result for the general case is given again by (6.24) but with $\phi(x)$ in $V_{3}$ replaced by $\phi_{1}(x)$, and $d_{1 e}, k$ replaced by

$$
\begin{aligned}
d_{1 e}^{(1)} & =\sqrt{\left(\frac{L}{2 \chi_{0} \pi R \theta_{1}\left(1-\theta_{1}\right)}\right)}, \\
k_{1} & =1-2 \theta_{1} .
\end{aligned}
$$

For the case of a discrete spectrum one obtains

$$
f_{D}=-\frac{4}{3} d_{1 e}^{(1)} k_{1}-d_{1 e}^{(1)} \sum_{j=1}^{N} \frac{1}{\alpha_{j}}\left(r_{1 j} \Lambda\left(\alpha_{j}, \theta_{1}\right)+f D_{20} r_{2 j} \Pi\left(\alpha_{j}, \theta_{1}\right)\right)
$$

where

$$
\begin{aligned}
\Pi(\alpha, \theta) & =\Omega(\infty, \theta)-\Omega(\alpha, \theta) \\
& =\frac{\sin ^{2} \pi \theta}{2 \theta(1-\theta) \pi} \int_{-1}^{1} f(y)(1-y) \exp (-\alpha y) \int_{-1}^{x} \frac{\exp (\alpha x)(k-x) d x d y}{f(x)(1-x)} .
\end{aligned}
$$

This is plotted on Fig. 1 for various values of $\theta$. Note that in contrast to $\Lambda(\alpha, \theta)$ it does not tend to zero at small $\alpha$, that is at large velocities. One may check that (6.42) is identical to (6.36) by Taylor expansion of the viscoelastic terms in the factors involving $\theta_{1}$ in $d_{1 e}^{(1)}$.

It is of interest to compare this present discussion with a similar one in the final paragraphs of the corresponding section of [7].

From the relationship between the coefficients of the exponentials in $\varepsilon_{1}(x)$, $\varepsilon_{2}(x)$ and the physical viscoelastic coefficients [6] it may be deduced that the quantities $r_{i j} / \alpha_{j}, i, j=1,2$, are negative. This relies upon the fact that the ratios $D_{i j} / D_{10}, i, j=1,2$, are either positive or small enough not to influence the sign (that is proportional to $\kappa$ defined by (6.9)), which follows from (6.8), (6.12), (6.13), at least in various limiting cases. However, the quantity $D_{20}$ is negative, while $\Pi(\alpha, \theta)$ is positive. This means that the first term in the summation on the right of (6.42) is positive while the second term is negative. If, in fact, the summation term in (6.42) is due to hysteresis loss it is presumably true that the sum is always positive since on physical grounds the hysteretic component of friction must be positive. However, the present author has not been able to prove this in general. 


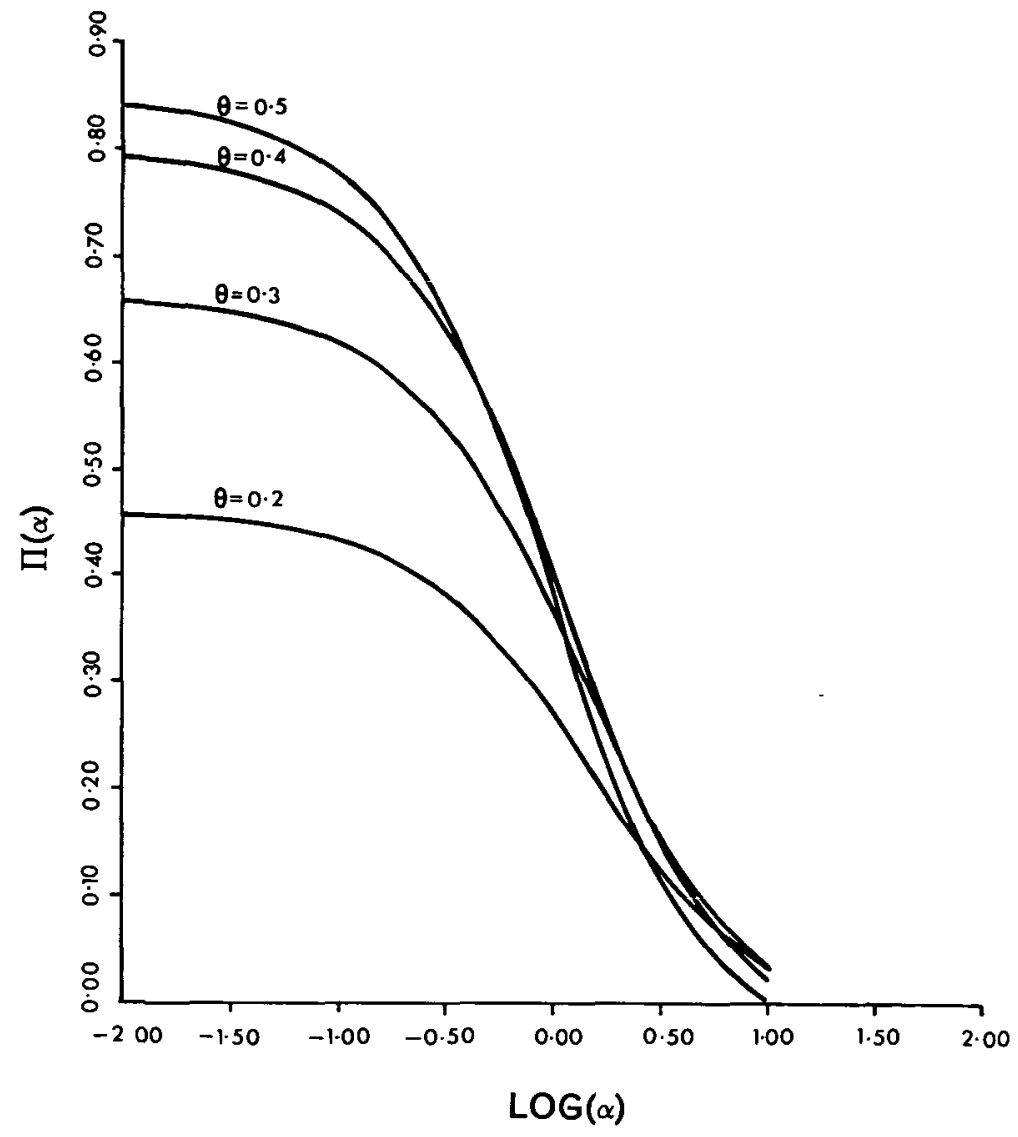

Fig. 1. Plot of the function $\Pi(\alpha, \theta)$ for various values of $\theta$.

It may be that a separation into hysteretic terms and others is simply not possible to achieve in general. From this viewpoint the summation term in (6.42) would represent the specifically hysteretic contribution only at very low velocities. Also the negative contribution in that term would present no problem in principle.

Note that the last term in (6.36) is definitely positive.

In Fig. $2, f_{D} / d_{1 e}$ is plotted for various values of $\theta$, together with the corresponding curves neglecting inertial effects. The independent variable is the ratio of velocity to the speed of transverse waves in the elastic limit. The input friction values range from 0.1 to 5.0 while Poisson's ratio in the elastic limit was taken to be 0.4 . The corresponding values of $\theta$ are shown. Only one term in the summation is included with the ratio of elastic to viscoelastic coefficient in $\mu(t), \lambda(t)$ respectively taken to be $0.1,0.05$. The two time constants are taken to be equal while the dimensionless combination $(b-a) /\left(2 c_{T}^{(e)} \tau\right)$, where $\tau$ is the common time constant, is given a value 0.1 . The agreement between the non-inertial approximations and 


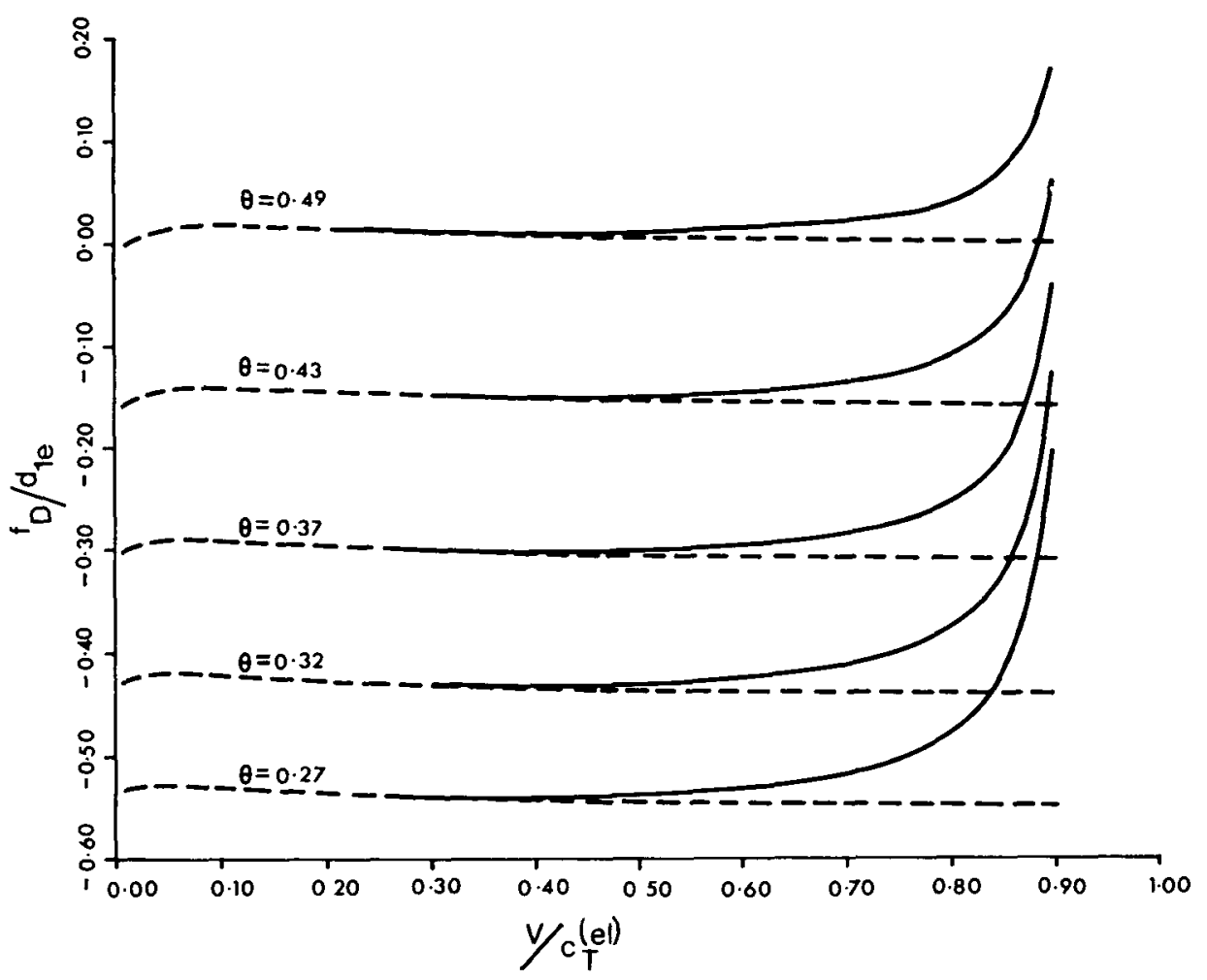

Fig. 2. Plot of the quantity $f_{D} / d_{10}$ given by (6.36) for a range of input friction values. See text for details of input parameters. Broken lines give non-inertial behaviour.

the more exact curves is good up to velocities of about $\frac{1}{3} c_{T}^{(e l)}$. Above this the non-inertial curves decrease slowly while the inertial curves increase and in fact become infinite at $V=c_{T}^{(e l)}$. This singularity is, however, probably an aberration resulting from the approximation made rather than a reflection of a real physical breakdown in the system. More specifically, it is clearly invalid to expand about the elastic limits of, for example, $A_{2}$ given by $(2.20)$, as $V / c_{T}^{(e l)} \rightarrow 1$. One would perhaps expect a peak in the curves in this limit but with an actual singularity avoided by the presence of small imaginary terms in $c_{T}(\omega)$ due to viscoelasticity. There is no singularity in the first term on the right of (6.42) which is consistent with the above discussion. This term is negative, which, as pointed out in [7], can cause $f_{D}$ as a whole to be negative.

The formulae given in this section and indeed in the paper as a whole are valid for velocities below $c_{T}^{(e l)}$ and break down as this value is approached. The question of how they must be modified to apply to the supersonic case will not be examined here. 


\section{Conclusions}

It has been shown that the method developed in $[6,7]$ can be extended to include inertial effects at least in an approximate sense. The formalism has been worked out in detail to first order in inertial terms with viscoelastic terms fully included, but where bulk and shear viscoelastic functions are assumed to be proportional. The resulting equations are substantially more complex than the corresponding ones in [7]. For the case of a discrete spectrum and a smooth punch, the detailed equations are given by (4.21), (4.26), the latter referring to (2.58), (2.61). For a cylindrical punch the important relations are (5.15)-(5.18).

As in previous papers, an approximate formalism is also worked out based on the assumption that viscoelastic effects are small compared with elastic effects. As before, this makes it possible to give explicit expressions for all quantities of interest. Inertial effects are incorporated exactly in these results. In particular the deformation coefficient of friction is given by (6.24) in the general case and (6.36), (6.41) for a discrete spectrum.

\section{Acknowledgements}

I wish to thank P. J. O'Keeffe, Head of Roads Division, and Dr. A. J. Curran, Head of Road Safety Section, National Institute for Physical Planning and Construction Research, for their help and encouragement.

\section{Appendix}

In Appendix 2 of [7] integrals of the type

$$
H(y, \theta)=\frac{1}{\pi} \int_{-1}^{1} \frac{q(x) f(x)}{x-y} d x
$$

were evaluated, where $q(x)$ is a polynomial of degree $m$ and $f(x)$ is given by (2.54). In Appendix 2 of [6] an integral closely related to $H\left(y, \frac{1}{2}\right)$ was evaluated. An alternative and more elegant method of handling this special case is to expand $q(x)$ in terms of Chebyshev polynomials and use the results of, for example [5]. $\dagger$ This approach will be generalized here to handle $H(y, \theta)$. The orthogonal polynomials corresponding to the weight function $f(x)$ are the Jacobi polynomials $P_{n}^{(-\theta, \theta)}(x)$ (see [2]). The polynomial $q(x)$ may be expanded in terms of these:

$$
q(x)=\sum_{n=0}^{m} c_{n} P_{n}^{(-\theta, \theta)}(x)
$$

† I wish to thank A. H. England for drawing my attention to this point. 
where [8]

$$
c_{n}=\frac{\left(n+\frac{1}{2}\right)(n !)^{2}}{\Gamma(1+n-\theta) \Gamma(1+n+\theta)} \int_{-1}^{1} P_{n}^{(-\theta, \theta)}(x) q(x) f(x) d x .
$$

This may easily be evaluated in general if $q(x)$ is expressed in the form

$$
q(x)=\sum_{r=0}^{m} \kappa_{r}(1+x)^{r}
$$

and the integral [2]

$$
\int_{-1}^{1} P_{n}^{(-\theta, \theta)}(x) f(x)(1+x)^{r} d x=\frac{2^{r+1} \Gamma(\theta+r+1) \Gamma(n-\theta+1) r !}{(r-n) !(r+n+1) !}
$$

is used. Note that this is zero if $r<n$. Using the integral [3]

$$
\begin{aligned}
\frac{1}{\pi} \int_{-1}^{1} \frac{f(x) P_{n}^{(-\theta, \theta)}(x)}{x-y} & =-\frac{2}{\pi} g(y) Q_{n}^{(-\theta, \theta)}(y), \quad|y|>1 \\
& =-\frac{2}{\pi} f(y) Q_{n}^{(-\theta, \theta)}(y), \quad|y|<1,
\end{aligned}
$$

where $g(y)$ is given by $(2.54)$ and $Q_{n}^{(-\theta, \theta)}(y)$ is a Jacobi function of the second kind [2], one has that

$$
\begin{aligned}
H(y, \theta) & =-\frac{2}{\pi} \sum_{n=0}^{m} c_{n} g(y) Q_{n}^{(-\theta, \theta)}(y), \quad|y|>1 \\
& =-\frac{2}{\pi} \sum_{n=0}^{m} c_{n} f(y) Q_{n}^{(-\theta, \theta)}(y), \quad|y|<1 .
\end{aligned}
$$

The function $P_{n}^{(-\theta, \theta)}(x)$ is given explicitly by [2]:

$$
P_{n}^{(-\theta, \theta)}(x)=\frac{1}{2^{n}} \sum_{r=0}^{n}\left(\begin{array}{c}
n-\theta \\
r
\end{array}\right)\left(\begin{array}{c}
n+\theta \\
n-r
\end{array}\right)(x-1)^{n-r}(x+1)^{r}
$$

so that, for example,

$$
\begin{aligned}
& P_{0}^{(-\theta, \theta)}(x)=1, \\
& P_{1}^{(-\theta, \theta)}(x)=x-\theta,
\end{aligned}
$$

and so on. The function $Q_{n}^{(-\theta, \theta)}(x)$ is given explicitly by [2]:

$$
\begin{aligned}
Q_{n}^{(-\theta, \theta)}(x)= & \frac{\pi}{2 \sin \pi \theta} P_{n}^{(-\theta, \theta)}(x)+\frac{1}{2} \frac{\Gamma(-\theta) \Gamma(n+\theta+1)}{n ! g(x)} \\
& \times F\left(n+1,-n, 1+\theta, \frac{1}{2}(1-x)\right), \quad|x|>1,
\end{aligned}
$$


where $F(a, b, c, z)$ is the standard hypergeometric function. However, also from [2] one has

$$
\begin{aligned}
P_{n}^{(\theta,-\theta)}(x) & =(-1)^{n} P^{(-\theta, \theta)}(-x) \\
& =\frac{\Gamma(n+\theta+1)}{\Gamma(1+\theta) n !} F\left(n+1,-n, 1+\theta, \frac{1}{2}(1-x)\right),
\end{aligned}
$$

giving

$$
Q_{n}^{(-\theta, \theta)}(x)=\frac{\pi}{2 \sin \pi \theta}\left[P_{n}^{(-\theta, \theta)}(x)+\frac{(-1)^{n+1} P^{(-\theta, \theta)}(-x)}{g(x)}\right]
$$

It follows from (A.7) that

$$
H(y, \theta)=-\frac{1}{\sin \pi \theta}(q(y) g(y)-B(y)), \quad|y|>1,
$$

where

$$
B(y)=\sum_{n=0}^{m}(-1)^{n} c_{n} P_{n}^{(-\theta, \theta)}(-x)
$$

One deduces that

$$
H(y, \theta)=-\frac{1}{\sin \pi \theta}(q(y) \cos \pi \theta f(y)-B(y)), \quad y|<| .
$$

These results are in some respects more explicit and convenient than those of Appendix 2 of [7].

\section{References}

[1] G. Eason, "The stresses produced in a semi-infinite solid by a moving surface force", Int. J. Engng. Sci. 2 (1965), 581-609.

[2] A. Erdelyi (ed.), Higher transcendental functions, Vol. 2, Bateman Manuscript Project (McGraw-Hill, New York, 1954), Chapter 6.

[3] A. Erdelyi (ed.), Tables of integral transforms, Vol. 2, Bateman Manuscript Project (McGraw-Hill, New York, 1954).

[4] L. A. Galin, Contact problems in the theory of elasticity, translated by H. Moss, I. N. Sneddon (ed.) (Department of Mathematics, North Carolina State College), Chapter 1.

[5] G. M. Gladwell and A. H. England, "Orthogonal polynomial solutions to some mixed boundary-value problems in elasticity theory", Quart. J. Mech. Appl. Math. 30 (1977), $175-185$.

[6] J. M. Golden, "Hysteretic friction of a plane punch on a half-plane with arbitrary viscoelastic behaviour", Quart. J. Mech. Appl. Math. 30 (1977), 23-49.

[7] J. M. Golden, "The problem of a moving rigid punch on an unlubricated viscoelastic half-plane", Quart. J. Mech. Appl. Math. 32 (1979), 25-52. 
[8] I. S. Gradshteyn and I. M. Ryzhik, Tables of integrals, series and products (Academic Press, New York and London, 1965).

[9] A. E. H. Love, A treatise on the mathematical theory of elasticity (Cambridge University Press, 1934), Chapter 10.

[10] I. N. Sneddon, Fourier transforms (McGraw-Hill, New York, 1951), Chapter 1.

[11] F. G. Tricomi, Integral equations (Interscience Publishers Inc., New York, 1957), Chapter 4.

\section{Road Safety Section \\ National Institute for Physical Planning and Construction Research \\ Waterloo Road \\ Dublin 4 \\ Ireland}

